\title{
ENDOCRINE THERAPY INTAKE AND OVERALL SURVIVAL IN YOUNG WOMEN WITH BREAST CANCER
}

Alessandra Borba Anton de Souza', Beatriz Fetzner ${ }^{1}$, Ester da Rosa', Gustavo Roesler ${ }^{1}$, Isabela Albuquerque Severo de Miranda1, Marcelle Morais dos Santos¹, Felipe Pereira Zerwes¹, Antônio Luiz Frasson¹

'Pontifícia Universidade Católica do Rio Grande do Sul - Porto Alegre (RS), Brazil.

Objective: Breast cancer (BC) in young women with positive hormone receptors $(\mathrm{HR}+)$ has a poor prognosis. There are possible clinical and biological explanations for these findings, being partially attributed to suboptimal adherence to endocrine therapy (ET). Fertility is one of the key factors for treatment discontinuation. This study aims to estimate the adherence of ET and the overall survival (OS) rate of BC/HR+ in young patients. Methods: We identified women from the public health system, diagnosed with stage I-III BC presenting at one single BC Center, between January 2006 and December 2015. Using the medical records of the hospital database, we constituted a cohort of 74 women aged $\leq 40$ years. The discontinuation rate with associated factors and OS were summarized as percentages. Results: A total of 51 women were $\mathrm{BC} / \mathrm{HR}+$. The mean age at diagnosis was 35 years. The median follow-up was 89 months. Among them, $45 \%$ had BC recurrence (local and/or distance), and $21 \%$ died. A total of $15 \%$ of patients interrupted the ET. The reasons for interruption were pregnancy (three patients), menstrual disorders (two patients), and irregular adherence (three patients). Tamoxifen (TMX) was prescribed in $74 \%$ of cases. About $19 \%$ switched their treatment to aromatase inhibitors. The genetic risk assessment was recommended to $58 \%$ of patients, $13 \%$ performed genetic tests, and $2 \%$ of patients carried out the pathogenic mutation in the high-risk BC genes. Conclusion: This cohort showed $84 \%$ of ET intake should be improved. Pregnancy issues and irregular adherence were the main reasons for discontinuation. Considerations of $78 \%$ OS in 7 years are that TMX was the only ET for most of the cohort, and it is considered undertreatment according to the current recommendations, and the low rate of genetic tests performed leads unrecognized high risk of potential recurrence in women with hereditary BC. Research in medical records should be addressed as a limitation.

Keywords: Young Adult; Women, Breast Neoplasms; Medication Adherence; Hormone Therapy; Tamoxifen. 